OPEN ACCESS

Edited by: Carlos Enrich,

University of Barcelona, Spain

Reviewed by:

Vas Ponnambalam,

University of Leeds, United Kingdom

Duarte C. Barral,

Universidade Nova de Lisboa,

Portugal

*Correspondence:

Wei Li

liwei@bch.com.cn

${ }^{t}$ These authors have contributed equally to this work

Specialty section: This article was submitted to

Membrane Traffic,

a section of the journal Frontiers in Cell and Developmental

Biology

Received: 17 July 2021 Accepted: 22 December 2021 Published: 17 February 2022

Citation:

Lu J, Ma J, Hao Z and Li W (2022) HPS6 Regulates the Biogenesis of Weibel-Palade Body in Endothelial Cells Through Trafficking v-ATPase to

Its Limiting Membrane.

Front. Cell Dev. Biol. 9:743124. doi: 10.3389/fcell.2021.743124

\section{HPS6 Regulates the Biogenesis of Weibel-Palade Body in Endothelial Cells Through Trafficking v-ATPase to Its Limiting Membrane}

\author{
Jiran $\mathrm{Lu}^{\dagger}$, Jing $\mathrm{Ma}^{\dagger}$, Zhenhua Hao and Wei $\mathrm{Li}^{*}$ \\ Beijing Key Laboratory for Genetics of Birth Defects, MOE Key Laboratory of Major Diseases in Children, Rare Disease Center, \\ National Center for Children's Health, Beijing Children's Hospital, Beijing Pediatric Research Institute, Capital Medical University, \\ Beijing, China
}

The Weibel-Palade body (WPB) is one of the lysosome-related organelles (LROs) in endothelial cells, whose main content is von Willebrand factor (VWF). The biogenesis of LROs is regulated by the Hermansky-Pudlak syndrome (HPS) protein-associated complexes through transporting cargo proteins to WPBs. Our previous studies have shown that HPS6, a subunit of BLOC-2 complex, is likely involved in the maturation of WPBs. However, the underlying mechanism remains unknown. In this study, we found that the knockdown of HPS6 in human umbilical vein endothelial cells (HUVECs) resulted in misshaped WPBs, decreased WPB number, and impaired VWF tubulation, which are similar to the characteristics of HPS6-deficient mouse endothelial cells. We observed similar morphological changes of WPBs in HUVECs after the knockdown of ATP6VOD1 (a subunit of V-ATPase). Furthermore, we found that HPS6 interacted with ATP6VOD1, suggesting that HPS6 transports ATP6VOD1 to the WPB limiting membrane for the assembly of the v-ATPase complex to maintain its acidic luminal $\mathrm{pH}$, which is critical for the formation of VWF tubules during WPB maturation. In conclusion, HPS6 likely regulates the biogenesis of WPBs by participating in the trafficking of $v$-ATPase to the WPB membrane.

Keywords: Weibel-Palade body, von Willebrand factor, lysosome-related organelle, Hermansky-Pudlak syndrome, HPS6, v-ATPase

\section{INTRODUCTION}

Endothelial cells (ECs) form a layer of flat and polygonal cells that line the vascular wall, which is the biological barrier between the circulating blood and the blood vessel wall and is of great significance to maintain vascular homeostasis (Aird, 2007). The Weibel-Palade body (WPB) is a type of lysosome-related organelles (LROs) (Marks et al., 2013), which was discovered in 1964 by Ewald Weibel and George Palade in rat and human ECs (Weibel and Palade, 1964; Warhol and Sweet, 1984). As a morphological marker of ECs, WPBs are rod-shaped granules with a diameter of 0.1-0.3 $\mu \mathrm{m}$ and a length of 1-5 $\mu \mathrm{m}$ (Arribas and Cutler, 2000; Michaux and Cutler, 2004; Valentijn et al., 2008). WPBs contain various bioactive molecules, such as von Willebrand factor (vWF), angiopoietin-2, interleukin-6 and -8, monocyte chemoattractant protein-1, and P-selectin, which are released in response to the activation of ECs (Schillemans et al., 2019). ECs play an important role in numerous physiological activities that include hemostasis, inflammation, angiogenesis, and wound 
healing. The main luminal content of WPB is vWF. vWF forms a highly multimerized tubular structure that is closely related to the shape and size of WPBs (Wagner et al., 1982; Karampini et al., 2020). Upon release into the plasma, the vWF tubules will unfurl into long strings that recruit platelets to prevent bleeding (Savage et al., 2002).

As a member of LROs, WPB biogenesis is likely regulated by Hermansky-Pudlak syndrome (HPS) protein-associated complexes (HPACs), which includes the biogenesis of lysosome-related organelles complex (BLOC)-1, -2, and -3, adaptor protein complex-3 (AP-3), and homotypic fusion and protein sorting complex (Wei and Li, 2013). It has been shown that AP-1/clathrin coat plays an essential role in forming WPBs in endothelial cells (Lui-Roberts et al., 2005) and that the recruitment of $\mathrm{CD} 63$ is dependent on AP-3 complex during the biogenesis of WPBs (Harrison-Lavoie et al., 2006). However, the functions of other HPACs in WPB biogenesis are unknown yet.

In our previous study ( $\mathrm{Ma}$ et al., 2016), we compared the phenotypes of WPBs and the secretion of vWF multimers in various HPAC mutant mice, including pa (HPS9 deficiency in BLOC-1), ru (HPS6 deficiency in BLOC-2), and ep (HPS1 deficiency in BLOC-3). We found that the regulated secretion of vWF in $r u$ mice was the most significantly reduced, and the maturation of WPBs in $r u$ mice was impaired significantly compared with that in wild-type (WT) mice. Therefore, we hypothesized that HPS6 may be required to deliver cargo proteins necessary for the biogenesis of WPBs.

In this study using $r u$ ECs and human umbilical vein endothelial cells (HUVECs), we firstly confirmed the impairments of WPB biogenesis and maturation due to HPS6 deficiency. Considering that neutralization treatment does not alter the elongated shape of WPBs in $r u$ ECs and vacuolar-type $\mathrm{H}^{+}$-ATPase (v-ATPase) is the primary proton pump for $\mathrm{H}^{+}$ homeostasis in organelles, we further found that HPS6 is required for the trafficking of subunit $\mathrm{d} 1$ in the $\mathrm{V} 0$ domain (ATP6V0D1) to WPBs to maintain the acidic luminal environment. Thus, HPS6 likely regulates the biogenesis of WPBs by transporting ATP6V0D1 to the WPB membrane to form highly ordered vWF tubules in the acidic lumen.

\section{RESULTS}

\section{Mutation of the Hps6 Gene in Mice Impairs WPB Biogenesis and Maturation}

Our previous study showed that WPBs in ru/Hps6 mutant mice lose their classical "cigar" shape and do not release enough vWF multimers into the plasma after desmopressin stimulation ( $\mathrm{Ma}$ et al., 2016). It is unknown whether these abnormities occur in the biogenesis stage or in the release stage. Therefore, we compared the shape and number of WPBs at various time points during their biogenesis between the primary ECs of WT and $r u$ mice. The cells were treated with phorbol-12myristate-13-acetate (PMA) to release existing mature WPBs. After washing out, the newly generated WPBs were examined at various time points $(0,2,4$, and $8 \mathrm{~h})$. Immunofluorescence staining and statistical analysis showed that the average number of WPBs per cell in $r u$ mice was higher than that in WT mice at 0-h time point after PMA treatment (Figures 1A,E) because there may be more immature WPBs that have not been released completely in $r u$ mice compared with WT mice. When treated for a longer time, more WPBs that represented nascent WPBs were seen in WT cells than in $r u$ cells. At $2 \mathrm{~h}$, the number of WPBs in WT and $r u$ ECs reached almost the same amount $(p>0.05)$. However, the number of WPBs in $r u$ ECs was significantly reduced at 8 -h time point compared with WT ECs (Figures 1B-E). The results of Feret's diameter of WPBs in WT and $r u$ mice showed a large cluster of WPBs in $r u$ mice whose Feret's diameter was concentrated at $0.5 \mu \mathrm{m}$ and then reached the highest percentage (nearly $30 \%$ ) at $2 \mathrm{~h}$ after PMA treatment. However, with the prolongation of time, WPBs in $r u$ cells with Feret's diameter of $\geq 1.3 \mu \mathrm{m}$ were rarely seen and WPBs with Feret's diameter of $\geq 1.8 \mu \mathrm{m}$ were only seen in WT cells (Figure 1F), which indicated less mature WPBs in $r u$ cells. Taken together, HPS6 deficiency may affect WPB biogenesis and maturation, which is consistent with our previous report (Ma et al., 2016).

\section{Biogenesis and Maturation of WPBs Are Compromised in HPS6 Knockdown HUVECs}

We further investigated the effects of HPS6 deficiency by siRNA knockdown of HPS6 (KD-HPS6) in HUVECs (Figure 2C). Immunofluorescence staining of $\mathrm{vWF}$ revealed more roundshaped WPBs in KD-HPS6 cells compared with negative control (NC) cells (Figures 2A,B). Moreover, the number of WPBs was significantly reduced in KD-HPS6 cells compared with NC cells (Figure 2D). These features resembled the changes in $r u$ mouse ECs as we reported previously (Ma et al., 2016).

Similarly, we observed the newly formed WPBs in HUVECs at various time points $(2,4,8$, and $16 \mathrm{~h})$ after PMA treatment (Figure 2E). Immunofluorescence staining and statistical analysis showed that the average number of WPBs per cell was significantly reduced in KD-HPS6 HUVECs at 4, 8, and $16 \mathrm{~h}$ after PMA treatment compared with the NC HUVECs (Figures 2E,F). In terms of the Feret's diameter of WPBs in these two groups, the statistical results showed that the distribution of Feret's diameter of WPBs in KD-HPS6 cells began to shift to small values from $4 \mathrm{~h}$ onward. At $16 \mathrm{~h}$, there were significantly more WPBs with a Feret's diameter of $\leq 1.5 \mu \mathrm{m}$ in KD-HPS6 cells, while many rod-shaped WPBs of $\geq 2.5 \mu \mathrm{m}$ were seen in NC cells (Figure 2G). These results confirmed that HPS6 deficiency affected the de novo production and maturation of WPBs.

\section{Regulated Secretion and Tubulation of vWF Are Compromised in HPS6 Knockdown HUVECs}

The existence of regular tubules of ultra-large vWF is an indicator of WPB maturation that is a driving force to form rod-shaped WPBs (McCormack et al., 2017; Tiemeier et al., 2020). Studies 


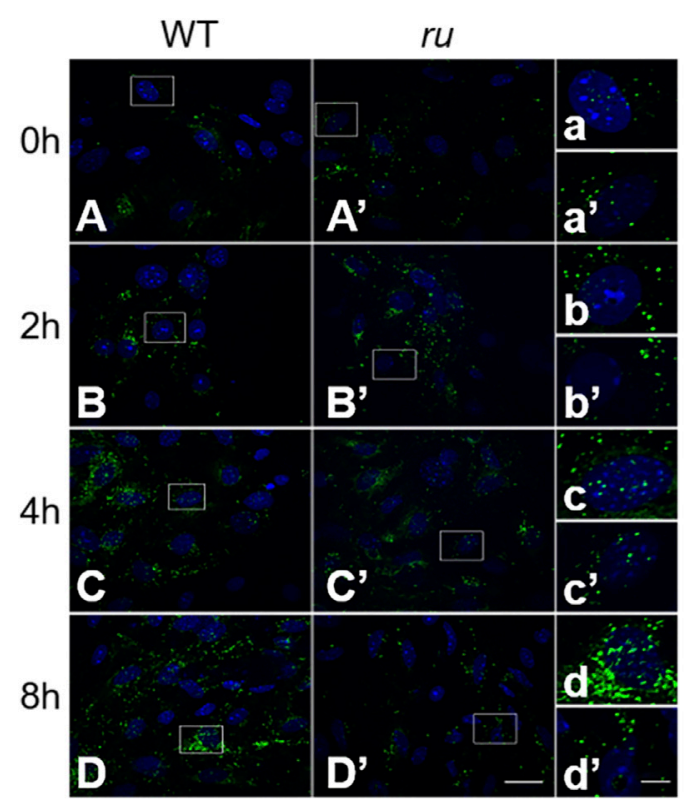

E

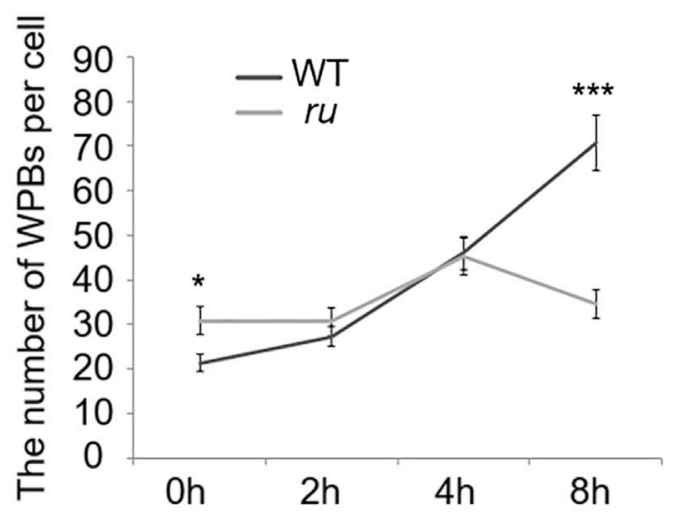

$\mathbf{F}$
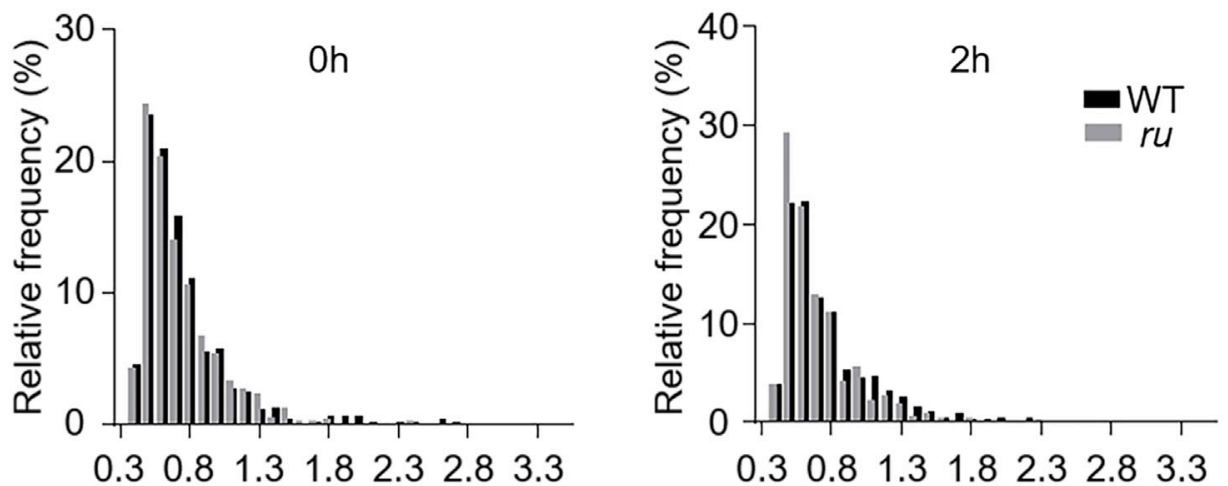

$\begin{array}{lllllll}0.3 & 0.8 & 1.3 & 1.8 & 2.3 & 2.8 & 3.3\end{array}$

$\begin{array}{lllllll}0.3 & 0.8 & 1.3 & 1.8 & 2.3 & 2.8 & 3.3\end{array}$

The Feret's diameters of WPBs $(\mu \mathrm{m})$ The Feret's diameters of WPBs $(\mu \mathrm{m})$
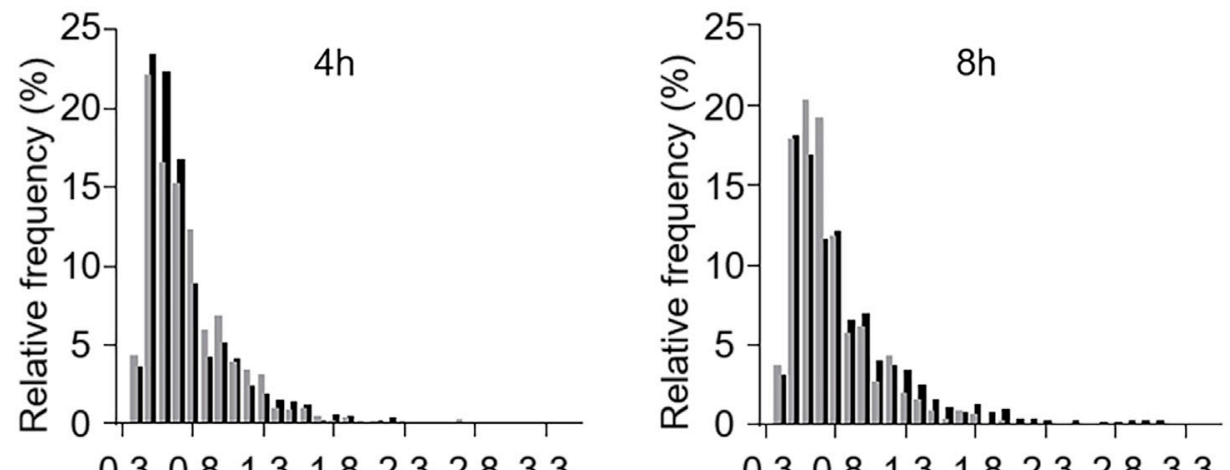

$\begin{array}{lllllll}0.3 & 0.8 & 1.3 & 1.8 & 2.3 & 2.8 & 3.3\end{array}$

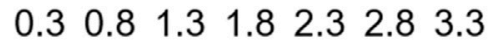

The Feret's diameters of WPBs $(\mu \mathrm{m})$ The Feret's diameters of WPBs $(\mu \mathrm{m})$

FIGURE 1 | The number and the elongated shape of newly formed Weibel-Palade bodies (WPBs) are affected in ru endothelial cells (ECs). Primary endothelial cells were isolated from 4-week-old male WT and ru mice and cultured for 10 days, then $80 \mathrm{nM}$ PMA was added to each dish to stimulate WPB secretion for 30 min, and the cells were fixed at 0, 2, 4, and $8 \mathrm{~h}$ after washing out. (A-D) $\left(\mathbf{A}^{\prime}-\mathbf{D}^{\prime}\right)$ Immunofluorescence images of primary endothelial cells at different time points labeled against vWF (green) and nucleus (DAPI, blue). Scale bar, $20 \mu \mathrm{m}$. The boxed square in $(\mathbf{A}-\mathbf{D})$ and $\left(\mathbf{A}^{\prime}-\mathbf{D}^{\prime}\right)$ was magnified as $(a-d)$ and $\left(a^{\prime}-d^{\prime}\right)$, respectively. Scale bar, $5 \mu \mathrm{m}$. (E) Quantitative analysis of the number of WPBs in each cell of WT and ru mice at different time points $\left(n=20,{ }^{\star} p<0.05,{ }^{\star \star *} p<0.001\right)$. (F) Feret's diameter distribution of

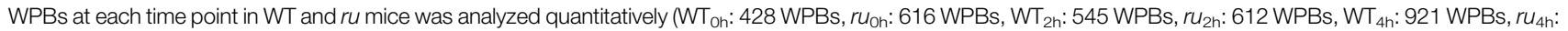
903 WPBs, $W_{8 n}: 1,412$ WPBs, and $r u_{8 h}: 692$ WPBs). All the images were analyzed by the NIH ImageJ software. Data were expressed as mean \pm SEM. Two independent experiments were performed. 

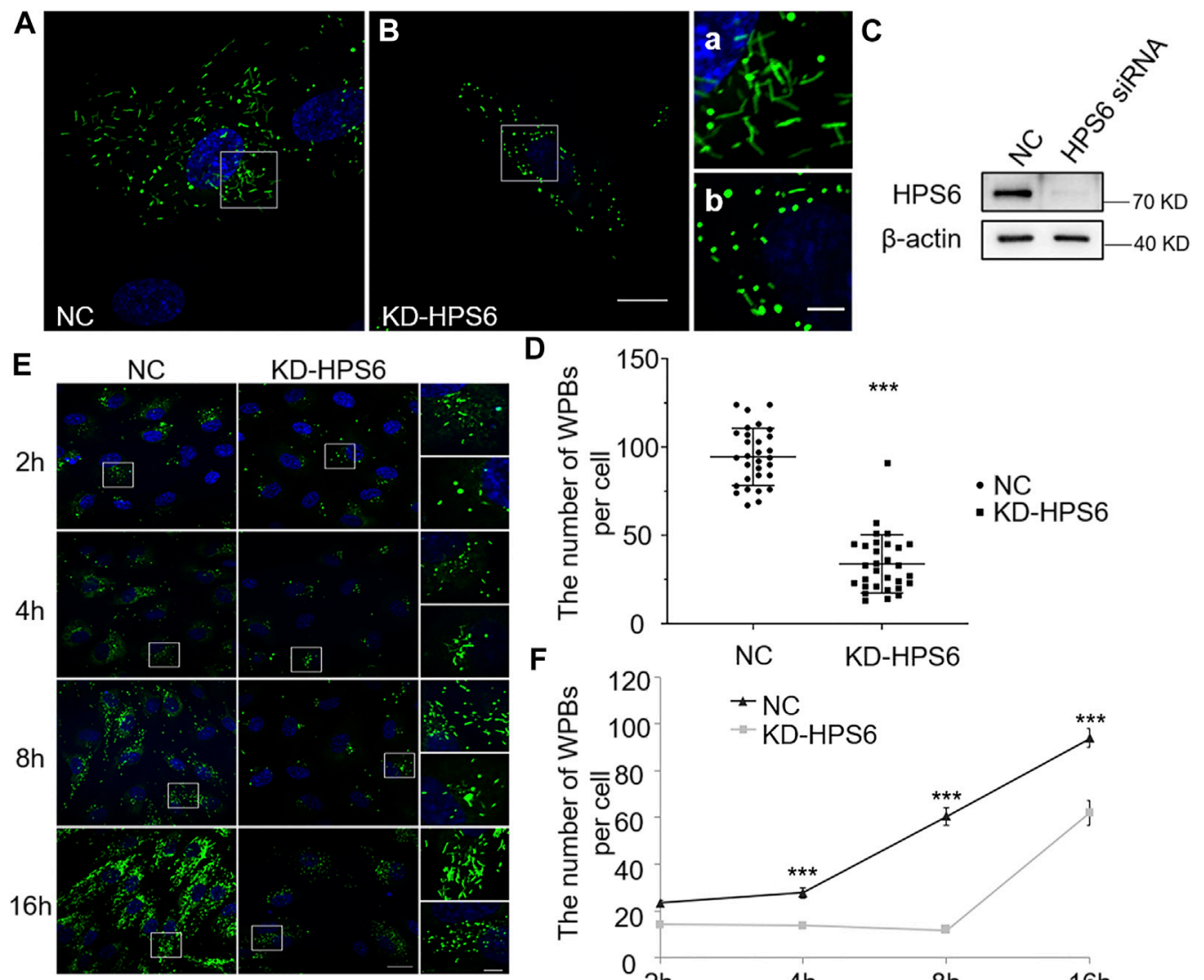

G
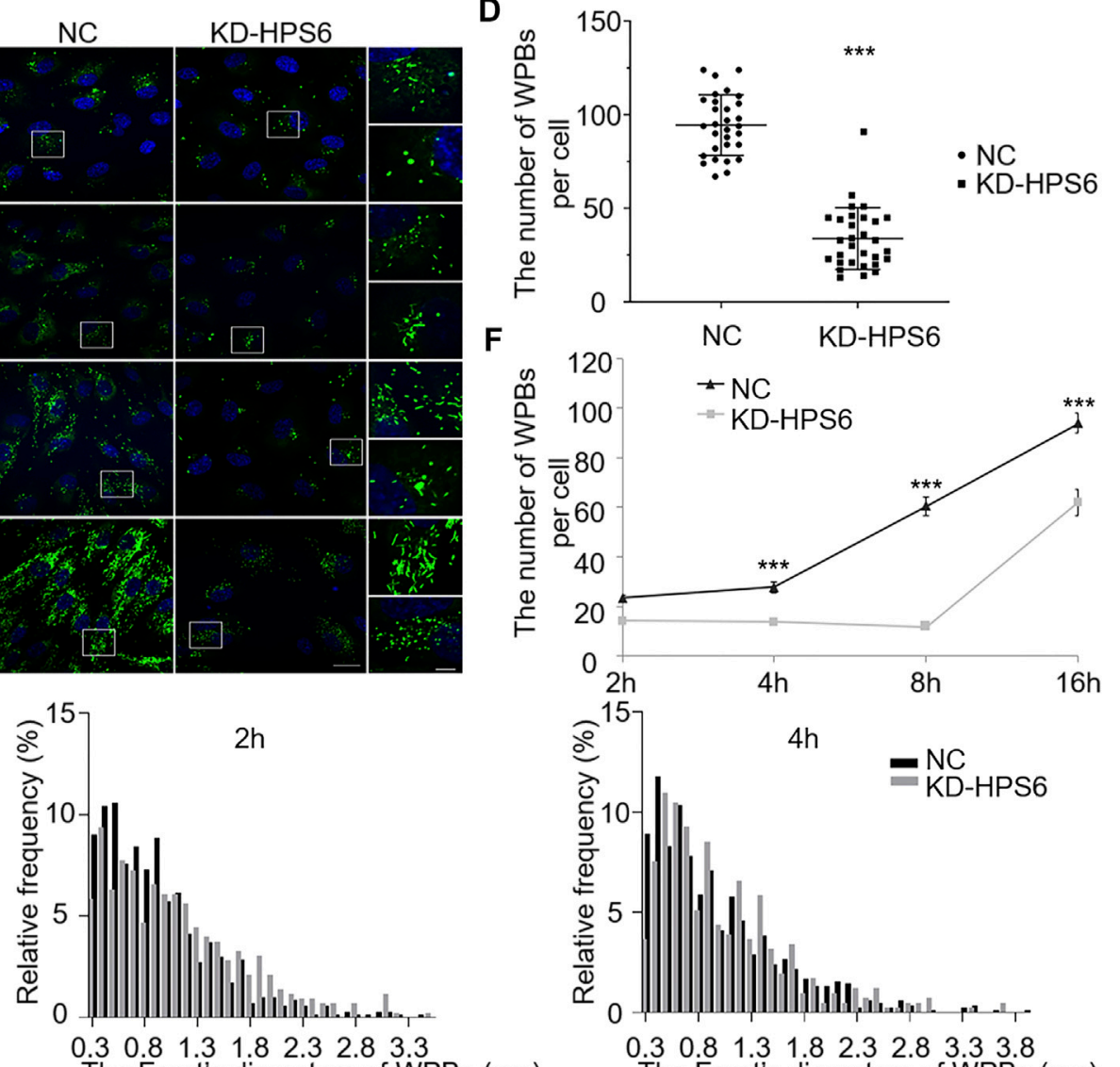

The Feret's diameters of WPBs $(\mu \mathrm{m})$
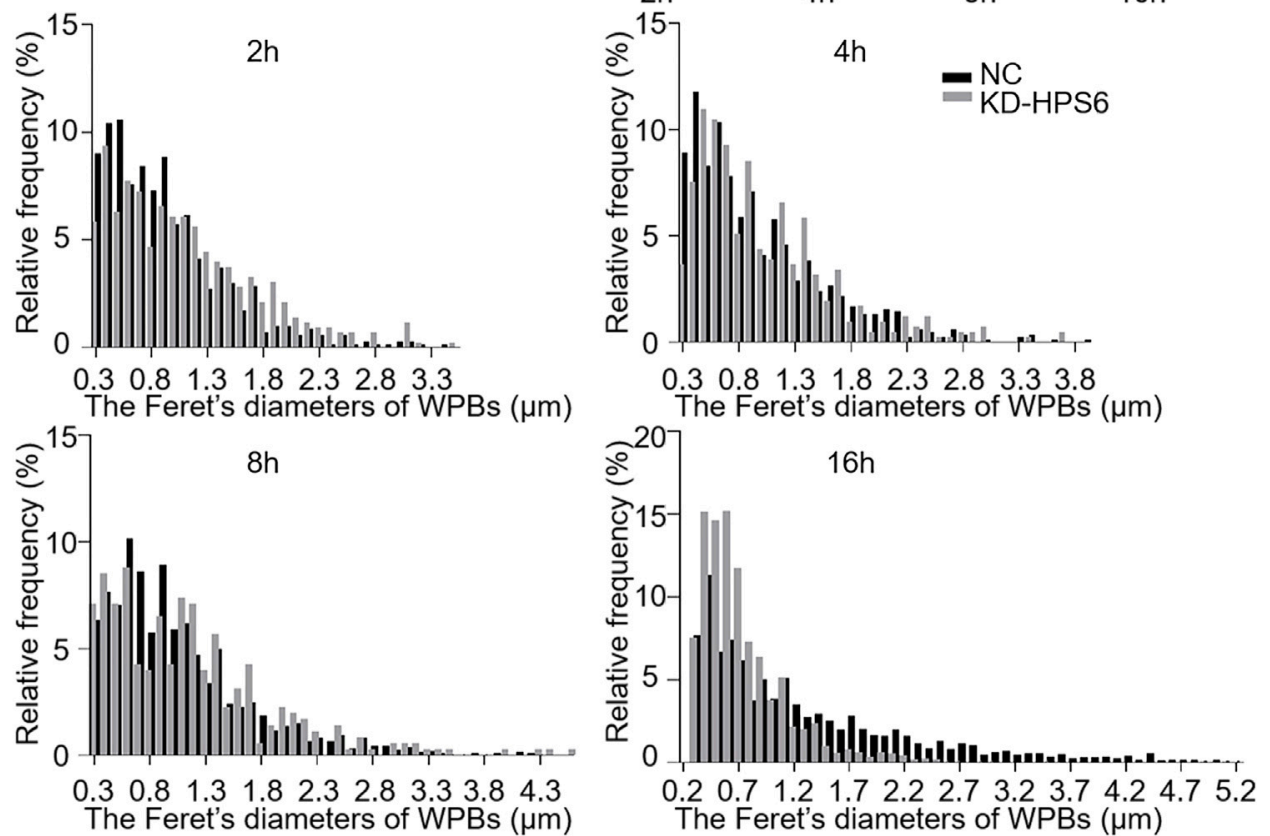

FIGURE 2 | The number and the elongated shape of newly formed Weibel-Palade bodies (WPBs) are affected in KD-HPS6 human umbilical vein endothelial cells (HUVECs). (A, B) Immunofluorescence images of negative control (NC; A) and HPS6 (KD-HPS6; B) siRNA-mediated knockdown in HUVECs labeled against vWF (green) and nucleus (DAPI, blue). Moreover, $80 \mathrm{nM}$ PMA was added to each dish to stimulate WPB secretion for $30 \mathrm{~min}$, and the cells were fixed at 2, 4, 8 , and $16 \mathrm{~h}$ after washing out. Scale bar, $20 \mu \mathrm{m}$. The boxed square in (A, B) was magnified as (a, b) respectively. Scale bar, $5 \mu \mathrm{m}$. (C) Western blotting analysis of the detection of HPS6 knockdown. (D) Quantitative analysis of the number of WPBs per cell of NC and KD-HS6 HUVECs $\left(n=30\right.$, $\left.{ }^{\star \star \star} p<0.001\right)$. (E) Both NC and KD-HPS6 HUVECs were exposed to 80 nM PMA for 30 min to stimulate WPB secretion, and the cells were fixed at 2, 4, 8, and 16 h, respectively, after washing out. Immunofluorescence images 
FIGURE 2 | showed the HUVECs at different time points labeled against vWF (green) and nucleus (DAPI, blue). Scale bar, 20 Hm. The boxed square was magnified, respectively. Scale bar, $5 \mu \mathrm{m}$. (F) Quantitative analysis of the number of WPBs per cell of NC and KD-HPS6 HUVECs $\left(n=20\right.$, $\left.{ }^{\star \star *} p<0.001\right)$. (G) Feret's diameter distribution of WPBs at each time point in NC and KD-HPS6 HUVECs was analyzed quantitatively $\left(\mathrm{NC}_{2 \mathrm{~h}}: 700\right.$ WPBs, KD-HPS6 $2 \mathrm{~h}: 427$ WPBs, NC $4 \mathrm{~h}$ : 831 WPBs, KDHPS6 $_{4 h}: 410$ WPBs, NC 8 h: 812 WPBs, KD-HPS6 8 h: 352 WPBs, NC Wh $_{16}$ 2,816 WPBs, and KD-HPS6 ${ }_{16 h}:$ 1,862 WPBs). Data were expressed as mean \pm SEM. Two independent experiments were performed.

have shown that vWF is released by regulated secretion and unfurls into long strings that are highly efficient in recruiting platelets under flow in neutral plasma (Michaux et al., 2006; Ferraro et al., 2014; El-Mansi and Nightingale, 2021). However, string formation is also dependent on the initial coiling of vWF into tubules. Disruption of coiling impairs orderly unfurling and generates tangles of multimers, which fail to be released into the plasma. WPBs lose their classical rod shapes after the knockdown of HPS6. We speculated that this might result in failure of vWF multimerization.

We used PMA as a stimulus to compare the regulated secretion of vWF multimers between NC and KD-HPS6 HUVECs. The deficiency of HPS6 was confirmed by Western blotting analysis in KD-HPS6 HUVECs (Figure 3A). Western blotting with an anti-vWF antibody was used to analyze the culture supernatant that was subjected to SDS-agarose gel electrophoresis. The results showed apparently regulated secretion in NC cells after PMA stimulation, but not in KDHPS6 cells (Figures 3B,E). These results suggested that HPS6 deficiency may lead to the failure of stimulated secretion of WPBs.

In general, VWF is secreted via three pathways. Regulated and basal secretion both occur from WPBs to transport highly multimerized VWF. Regulated secretion is triggered by agonist-mediated activation of the endothelium. In contrast, basal secretion occurs constitutively. The third pathway occurs by the constitutive release of VWF that has not been sorted into WPBs which is not subject to high multimerization. In Figure 3B, there was no difference between lane 1 (NC) and lane 3 (KDHPS6), which can be explained by the fact that the constitutive release and the basal secretion of vWF did not seem affected after KD-HPS6. However, the comparison of lanes 1 and 2 vs. lanes 3 and 4 showed that the regulated secretion of vWF was compromised. Decreased vWF secretion is also one of the reasons why HPS6 deficiency leads to coagulation disorders.

To analyze the tubulation of vWF, the ability to form vWF strings in NC and KD-HPS6 HUVECs was assessed after treatment with Triton X-100. Triton X-100 destroys cell membranes and WPB membranes, which exposed vWF to neutral pH in situ (Michaux et al., 2006), thereby causing in situ diffusion to form vWF strings. Immunofluorescence staining showed vWF strings in NC cells, but very rarely in KD-HPS6 cells (Figures 3C,D). In addition, we measured the length of vWF strings in each group of HUVECs and found that the vWF strings were much shorter in KD-HPS6 cells than in WT cells (Figure 3F). This suggests that $\mathrm{vWF}$ multimers failed to unfurl into the plasma from KD-HPS6 HUVECs after stimulation, indicating failure of tubule formation in these cells. Thus, HPS6 plays an important role in the tubulation and secretion of vWF multimers in WPBs.

\section{Steady-State Levels of Several Subunits of v-ATPase Complex Are Decreased in HPS6 Knockdown Cells}

As vWF tubulation requires an appropriate acidic environment, we reason that the misshaped WPBs and abnormal release of WPBs in KD-HPS6 HUVECs might be due to a change of the $\mathrm{pH}$ value in the lumen of WPBs. It is well known that $\mathrm{v}$-ATPase is the primary proton pump for $\mathrm{H}^{+}$homeostasis in organelles, which consists of two multi-subunit domains, V1 and V0. The V1 domain consists of $A, B, C, D, E, F, G$, and $H$ subunits, while the V0 domain consists of a, d, c, c", e, and Ac45 subunits. It binds and hydrolyzes ATP for active intermembrane transport of protons (Futai et al., 2019). Therefore, we treated HUVECs with bafilomycin A1 (Baf A1), a v-ATPase inhibitor (Tang et al., 2019), to observe whether the WPB shape and Feret's diameter changed accordingly. Our results showed that most WPBs in the Baf A1 group lost their elongated phenotype (Figure 4B), which was consistent with the phenotype of the KD-HPS6 group (Figure 2B), whereas no obvious abnormality was found in the DMSO control group (Figure 4A). The Feret's diameter distribution of WPBs in these two groups showed that more WPBs in the Baf A1 group had a Feret's diameter of $\leq 0.8 \mu \mathrm{m} \quad$ (Figure 4C), which indicated that v-ATPase inhibition significantly compromised the size of WPBs.

To further explore the effect of HPS6 on v-ATPase, the steadystate levels of several subunits of v-ATPase were examined in KDHPS6 HUVECs by Western blotting. We found that the expression levels of ATP6V0D1, ATP6V1H, and ATP6V1B2 in the KD-HPS6 group were significantly lower than those in the NC group (Figures 4D-I). Similarly, these changes were observed in HPS3 (another BLOC-2 subunit) knockdown HUVECs (data not shown). These results suggest that HPS6 deficiency may destabilize the v-ATPase complex in KD-HPS6 HUVECs, which likely results in a higher $\mathrm{pH}$ in the lumen of WPBs and disrupts vWF tubule formation.

\section{HPS6 Interacts With ATP6V0D1 for Its Trafficking to WPBs}

We next investigated whether v-ATPase subunits localized to WPBs. We observed a partial co-localization of the WPB marker vWF with Myc-tagged ATP6V0D1 (Figure 5A). To verify the subcellular localization, an Optiprep continuous density gradient was applied to examine the distribution of ATP6V0D1 and vWF in each fraction. The results showed that the main peak of vWF distribution was in the fractions 10-12, which may be the position of the mature WPBs, while the low-density fraction may represent the $\mathrm{vWF}$ in the ER, Golgi, and immature WPB. A proportion of ATP6V0D1 existed in the fractions 10-12, 

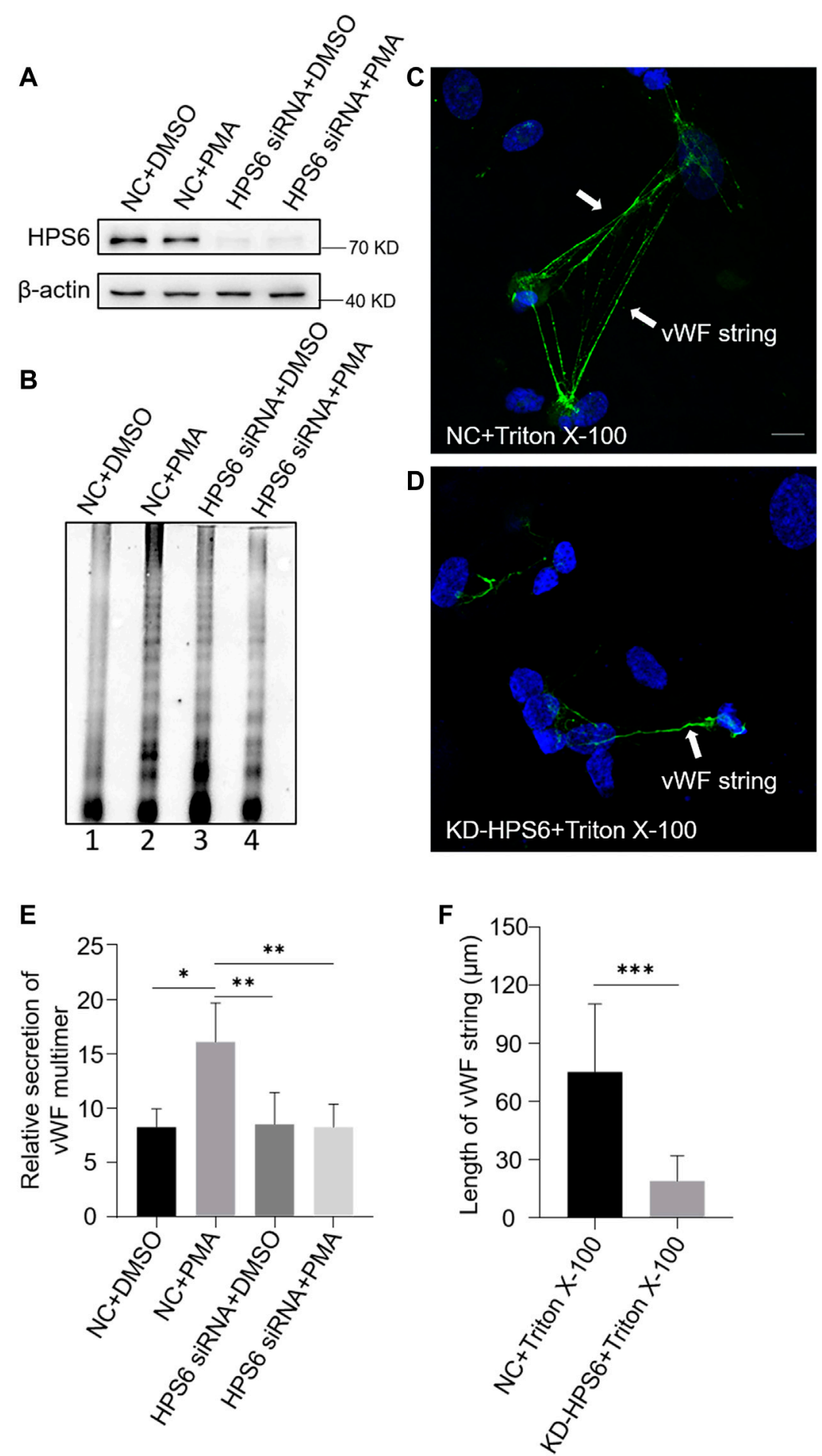

FIGURE 3 | von Willebrand factor (VWF) secretion and the ability to generate surface strings are compromised in KD-HPS6 human umbilical vein endothelial cells (HUVECs). The negative control (NC) and HPS6 siRNA were transfected into two groups of HUVECs, respectively. At 72 h later, one group of NC and KD-HPS6 cells was exposed to 80 nM PMA for 30 min to stimulate WPB secretion, and the other groups were exposed to $0.1 \%$ DMSO instead. (A) Western blotting analysis of the detection of HPS6 knockdown in cell lysate collection. (B, E) Western blotting analysis of vWF multimer secretion in supernatant. The multimer gels were analyzed using the $\mathrm{NIH}$ ImageJ software. The quantification of supernatant VWF multimers was carried out based on the normalization of the $\beta$-actin protein level of the cells in each well. $n=5,{ }^{\star} p<0.05,{ }^{\star \star} p<0.01$. (C, D) Moreover, $1 \%$ Triton X-100 was added into the culture medium of NC and KD-HPS6 HUVECs and cultured at $37^{\circ} \mathrm{C}$ for $1 \mathrm{~h}$. Immunofluorescence images of two groups of HUVECs labeled against VWF (green) and nucleus (DAPI, blue) were shown. Scale bar, $10 \mu \mathrm{m}$. (F) The length of vWF strings at each group of HUVECs was measured by the NIH ImageJ software $\left(n=30\right.$ per group, $\left.{ }^{\star \star \star} p<0.001\right)$. Data were expressed as mean \pm SEM. Three independent experiments were performed. 

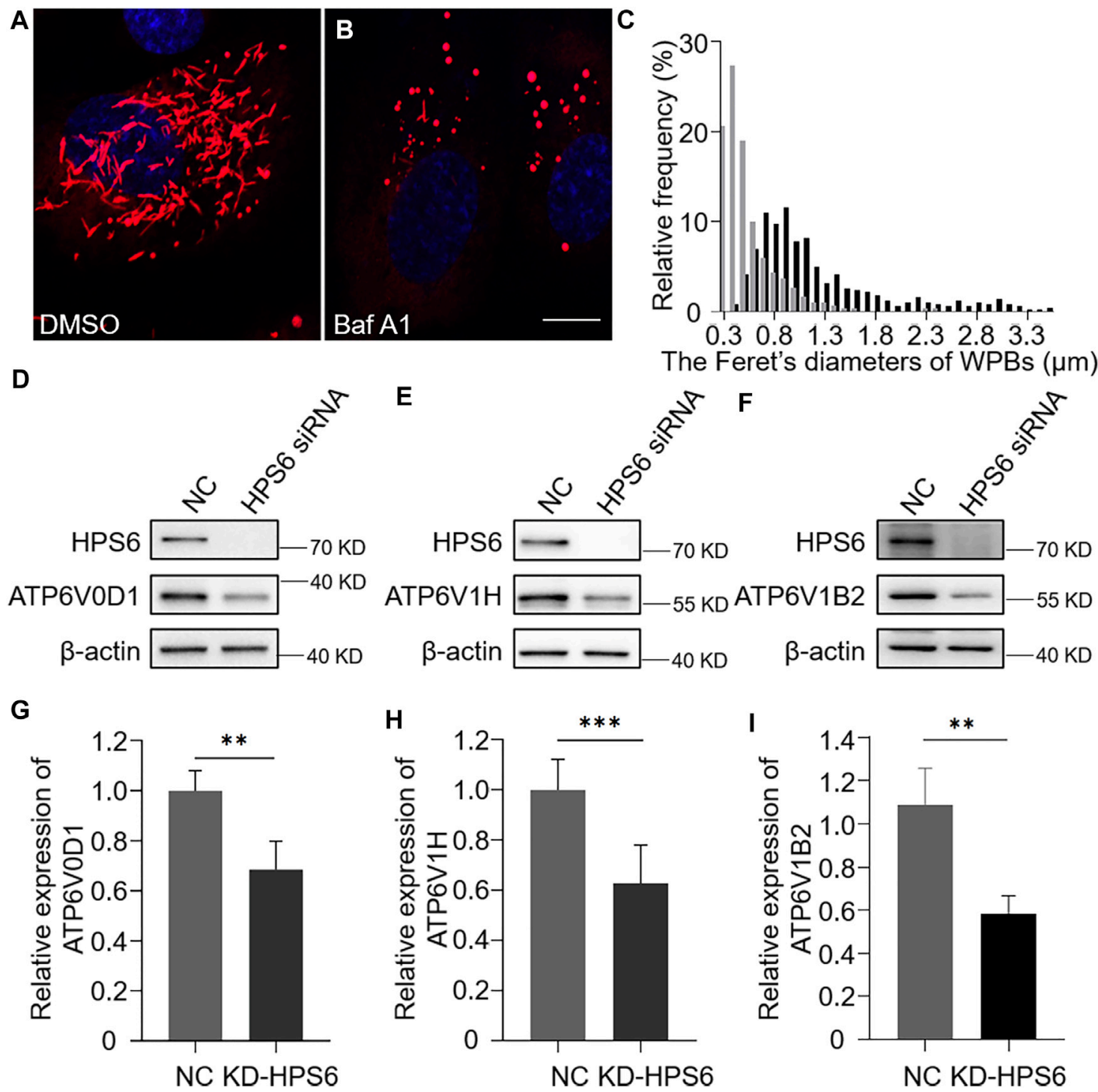

FIGURE 4 | v-ATPase is compromised in KD-HPS6 human umbilical vein endothelial cells (HUVECs). Pharmacological inhibition of the v-ATPase caused the Weibel-Palade bodies (WPBs) to lose their elongated shapes. DMSO or v-ATPase inhibitor, Baf A1 (200 nM), was administered $1 \mathrm{~h}$ before cell fixation by $4 \%$ PFA ( $n=20$ per group). (A, B) Immunofluorescence images of DMSO-treated (A) and Baf A1-treated cells labeled against vWF (red) and nucleus (DAPI, blue). Scale bar, 10 um. (C) Feret's diameter of WPBs at each group of HUVECs was analyzed quantitatively (DMSO: 502 WPBs, gray; Baf A1: 300 WPBs, black). (D-I) siRNA-mediated HPS6 knockdown suppressed the expression of ATP6V0D1, ATP6V1H, and ATP6V1B2, three subunits of V-ATPase $\left(n=8\right.$ per group, ${ }^{\star \star} p<0.01$, $\left.{ }^{\star \star \star} p<0.001\right)$. Data were expressed as mean \pm SEM. Three independent experiments were performed.

indicating that ATP6V0D1 is partially co-localized with mature WPBs. Part of ATP6V0D1 (fractions 5-8) was co-localized with late endosome/lysosome marker LAMP1 (Figure 5B). Furthermore, a co-immunoprecipitation analysis verified the interaction between Myc-tagged ATP6V0D1 and Flag-tagged HPS6 in $293 \mathrm{~T}$ cells (Figure 5C). Our endogenous immunoprecipitation (IP) also showed that HPS6 immunoprecipitated ATP6V0D1 (data not shown). These data suggest that ATP6V0D1 is likely transported to WPBs as a cargo of HPS6 or BLOC-2.

In order to compare the v-ATPase subunits on WPB after the knockdown of HPS6, we performed the Optiprep continuous density gradient experiment and took the same amount of fractions 10-12 that mainly represented the mature WPBs in NC and KD-HPS6 cells for Western blotting. The results showed that the protein levels of ATP6V0D1, ATP6V1H, and ATP6V1B2 were decreased in fractions 10-12 (Figure 5D). These results suggested that ATP6V0D1 could not be transported to the WPB membrane correctly after HPS6 deficiency, which likely affects the assembly of the v-ATPase complex and results in disrupted WPB acidification.

To explore whether the decrease in the protein level of ATP6V0D1 after HPS6 knockdown is due to the degradation of the mis-localized protein, we treated NC and KD-HPS6 
A
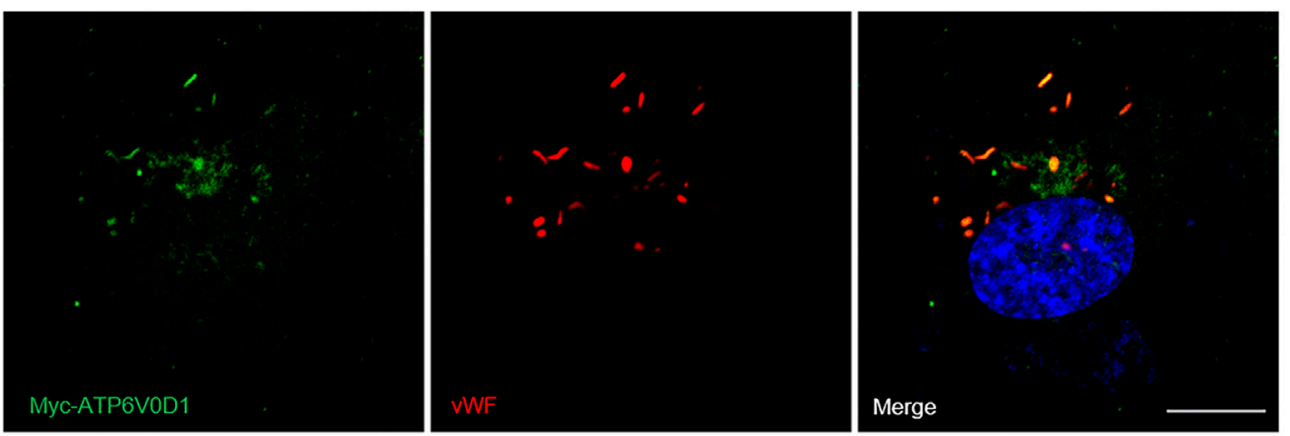
$50 \%$

Input $1 \begin{array}{llllllllllll}2 & 3 & 4 & 5 & 6 & 7 & 8 & 9 & 10 & 11 & 12 & 13\end{array}$

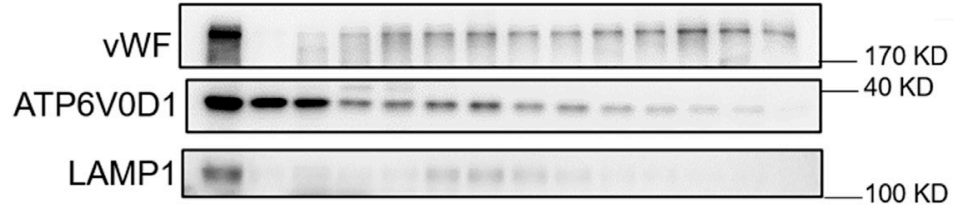

D

$$
\begin{aligned}
& \text { Input } \\
& 11 \quad 12 \\
& 121
\end{aligned}
$$
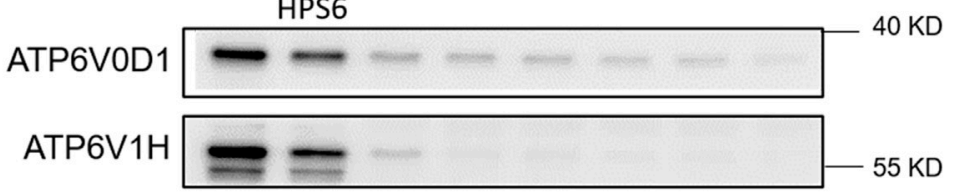

ATP6V1B2

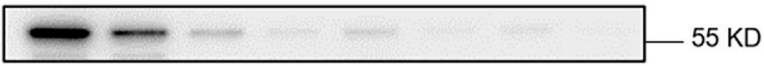

HPS6

$\beta$-actin

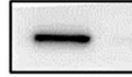

$-70 \mathrm{KD}$

G

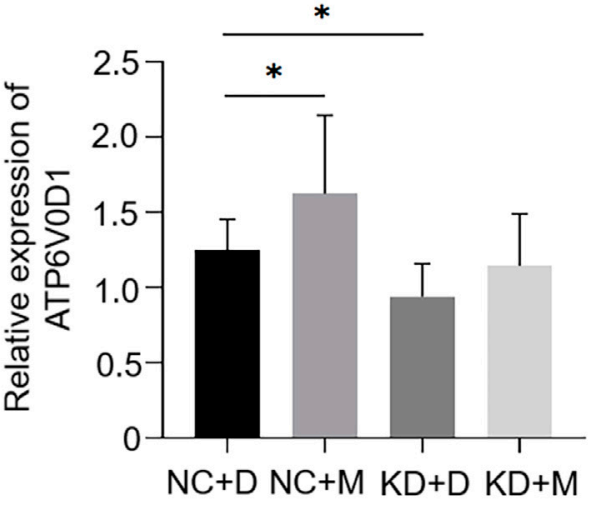

C

ATP6V0D1

Flag-HPS6

Flag-2B

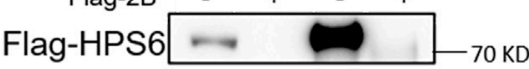

ATP

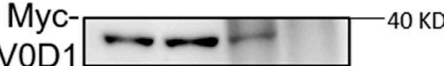

E

HPS6

ATP6V0D1

$\beta$-actin

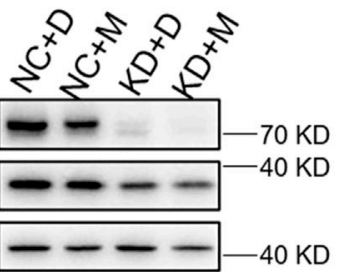

$\mathbf{F}$

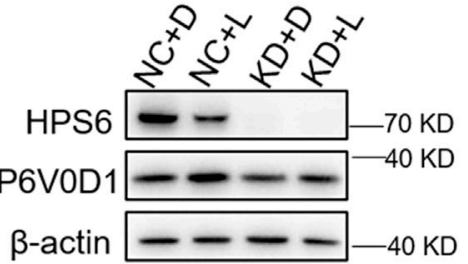

FIGURE 5 | HPS6 interacts with ATP6V0D1 and mediates its trafficking to the Weibel-Palade body (WPB). (A) Immunofluorescence images of Myc-ATP6VOD1 plasmid-transfected human umbilical vein endothelial cells (HUVECs) labeled against Myc (green), vWF (red), and nucleus (DAPI, blue). Scale bar, 10 um. (B) Separation of organelles and proteins by Optiprep density gradient. The final organelles and protein pellets were placed onto 50-5\% sucrose gradient layers and then centrifuged for $30,000 \mathrm{rpm}$ for $16 \mathrm{~h}$ at $4^{\circ} \mathrm{C}$. A total of 13 fractions were collected from the top to the bottom of the gradient for further Western blotting experiments to test the distribution of VWF and ATP6VOD1. (C) A total of 293 T cells were transfected with Flag-HPS6 and Myc-ATP6V0D1 plasmids, and the cell lysates were coimmunoprecipitated by anti-Flag M2 affinity gel. Western blotting analysis of detection of Flag and Myc bands. (D) NC and HPS6 siRNA were transfected into two groups 
FIGURE 5 | of HUVECs, respectively. At $72 \mathrm{~h}$ later, the organelles and proteins were separated by Optiprep density gradient. The input and fractions 10-12 of each group were analyzed by Western blotting. (E-H) The negative control (NC) and HPS6 siRNA were transfected into two groups of HUVECs, respectively. At 72 h later, one group of NC and KD-HPS6 cells was exposed to $0.25 \mu \mathrm{M}$ MG132 (M) or $20 \mu \mathrm{M}$ Leupeptin (L), and the other groups were exposed to $0.1 \%$ DMSO (D) instead. (E, G) Western blotting analysis of detection of ATP6V0D1 in KD + M cells. $(\mathbf{F}, \mathbf{H})$ Western blotting analysis of detection of ATP6V0D1 in KD $+\mathrm{L}$ cells. $n=7,{ }^{\star} p<0.05$, ${ }^{\star \star} p<0.01$. Data were expressed as mean \pm SEM. Two independent experiments were performed.

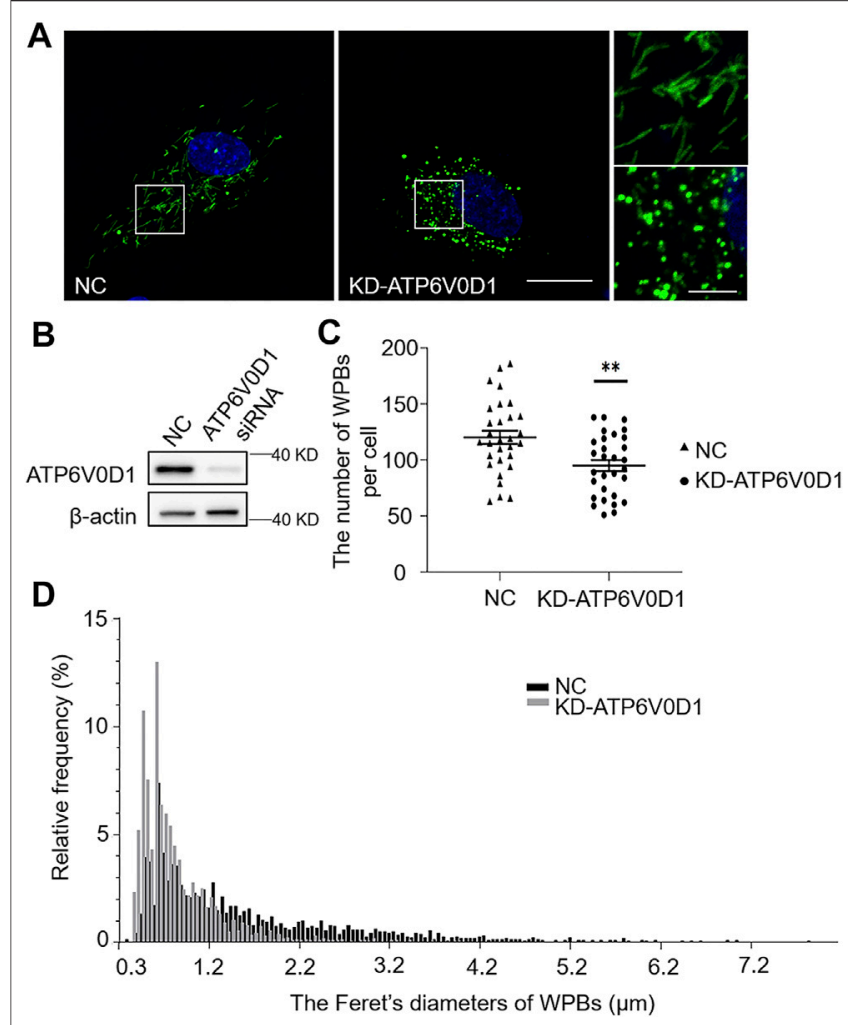

FIGURE 6 | Knockdown of ATP6VOD1 in human umbilical vein endothelial cells (HUVECs) phenocopies the abnormalities of Weibel-Palade bodies (WPBs) KD-HPS6 HUVECs. (A) Immunofluorescence images of negative control (NC) and ATP6VOD1 siRNA (KD-ATP6VOD1)-mediated knockdown in HUVECs labeled against VWF (green) and nucleus (DAPI, blue). Scale bar, $20 \mu \mathrm{m}$. The boxed squares were magnified, respectively. Scale bar, $5 \mu \mathrm{m}$. (B) Western blotting analysis of the detection of ATP6VOD1 knockdown. (C) Quantitative analysis of the number of WPBs per cell of NC and KD-ATP6VOD1 HUVECs ( $n=30,{ }^{* \star} p<0.01$ ). (D) Feret's diameter distribution of WPBs at each group of HUVECs was analyzed quantitatively (NC: 3,608 WPBs, black; KD-ATP6V0D1: 2,852 WPBs, gray). Data were expressed as mean \pm SEM. Three independent experiments were performed.

HUVECs with a proteasome inhibitor MG132 (M) (Mazzotta et al., 2020; Olmedo et al., 2020) and a lysosome inhibitor leupeptin (L) (Mazzotta et al., 2020), respectively. We optimized the concentration of $0.25 \mu \mathrm{M}$ MG132 and $20 \mu \mathrm{M}$ leupeptin as the experimental conditions. Our results showed that, although ATP6V0D1 was increased significantly in the $\mathrm{NC}+\mathrm{M}$ and $\mathrm{NC}+\mathrm{L}$ groups compared with that in the $\mathrm{NC}+\mathrm{D}$ (DMSO) group (Figures $\mathbf{5 E - H}$ ), a significant increase of ATP6V0D1 was found only in the KD $+\mathrm{L}$ group and not in the $\mathrm{KD}+\mathrm{M}$ group compared with the $\mathrm{KD}+\mathrm{D}$ group (Figures 5E-H). These results suggest that, although a portion of
ATP6V0D1 may be degraded by the proteosome, ATP6V0D1 is mainly subjected to lysosomal degradation, indicating that mis-targeted ATP6V0D1 is likely degraded via the lysosomal pathway that results in a decrease of the steady-state level of ATP6V0D1.

\section{ATP6V0D1 Knockdown Phenocopies the Abnormalities of WPBs in ru Mouse Cells and in KD-HPS6 HUVECs}

We next investigated the changes of WPBs in HUVECs after the knockdown of the ATP6V0D1 gene (KD-ATP6V0D1). We found that the changes in shape, number, and size of WPBs in KD-ATP6V0D1 HUVECs were similar to those in $r u$ mouse ECs and KD-HPS6 HUVECs (Figures 6A-D). The off-target effects of both siHPS6 and siATP6V0D1 were excluded by three different target siRNAs (data not shown). Additionally, the regulated secretion and ability to unfurl into the plasma of vWF multimers were impaired in KD-ATP6V0D1 HUVECs (Figure 7). These results further confirmed that HPS6 is likely involved in the trafficking of ATP6V0D1 to the WPB membrane for the assembly of v-ATPase to maintain the acidic $\mathrm{pH}$ in its lumen. This highlights the importance of $\mathrm{H}^{+}$homeostasis in the lumen of WPBs during their biogenesis and release.

\section{DISCUSSION}

The BLOC-2 complex functions in transporting cargos from endosomes to lysosomes or lysosome-related organelles in cells. Many studies have shown that the BLOC-2 complex plays an important role in the biogenesis of melanosomes (Dennis et al., 2015). As one of the subunits of the BLOC-2 complex, HPS6 has been reported to be involved in lysosome positioning and maturation in HeLa cells (Li et al., 2014). In this study, we found that HPS6 is involved in the trafficking of ATP6V0D1, a subunit of v-ATPase complex, to the WPB membrane for its assembly into $\mathrm{v}$-ATPase complex to maintain the acidic state of the WPB lumen, which is essential for vWF tubulation and the formation of elongated mature WPBs. When HPS6 is deficient, ATP6V0D1 is missorted and likely subjected to lysosomal degradation, which increases the $\mathrm{pH}$ in the lumen of WPBs and ultimately disrupts WPB biogenesis and secretion. This result explains one of the molecular mechanisms by which HPS6 participates in the biogenesis and maturation of WPBs.

Additionally, we revealed that the protein level of ATP6V1H and ATP6V1B2, two other subunits of v-ATPase, was significantly reduced in KD-HPS6 cells. v-ATPase is a macro- 


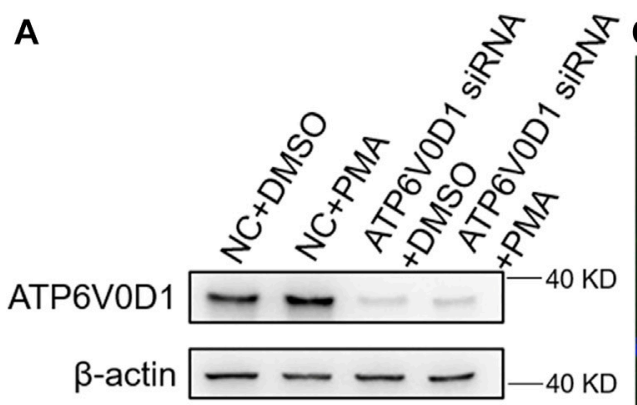

B
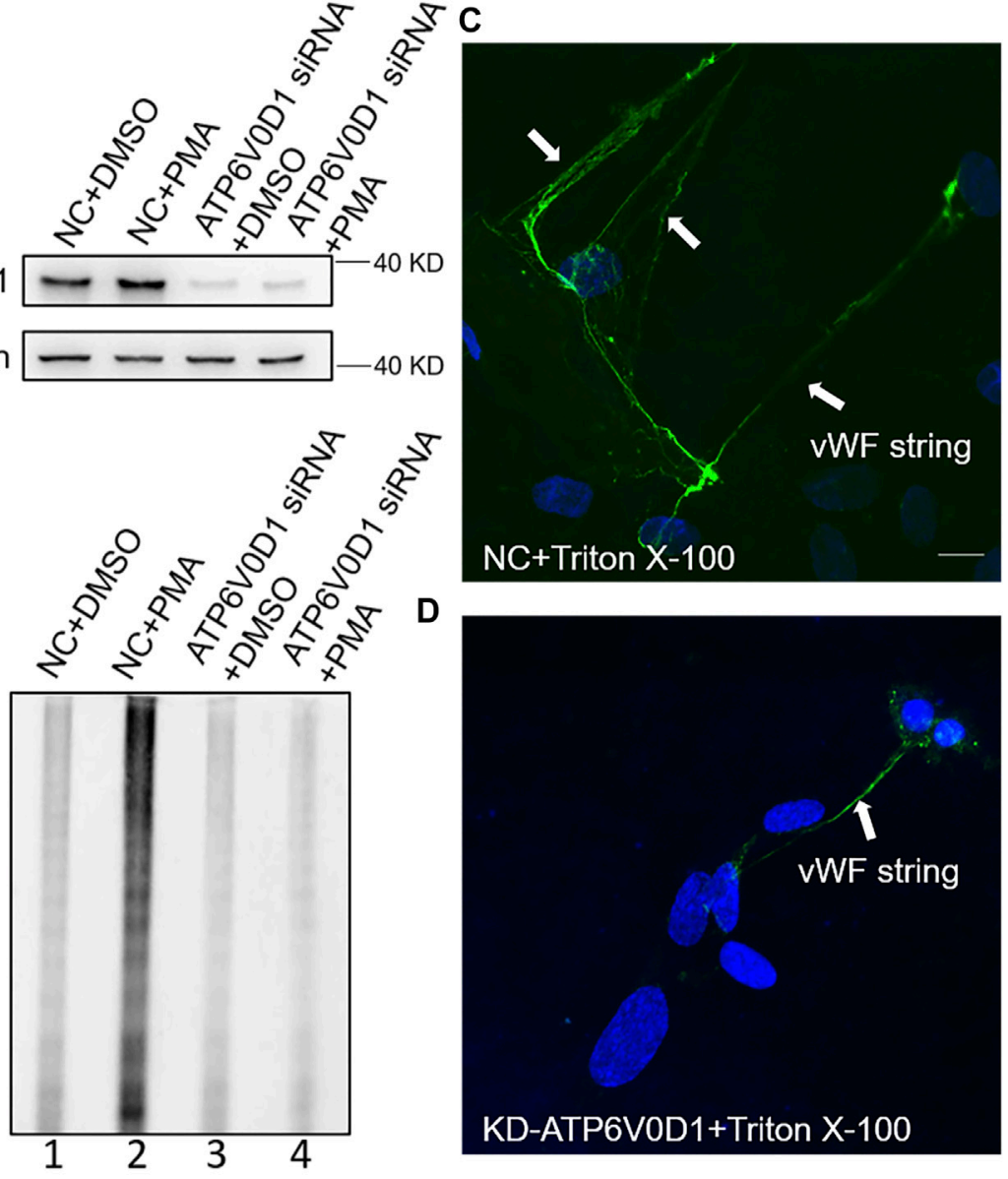

E

F
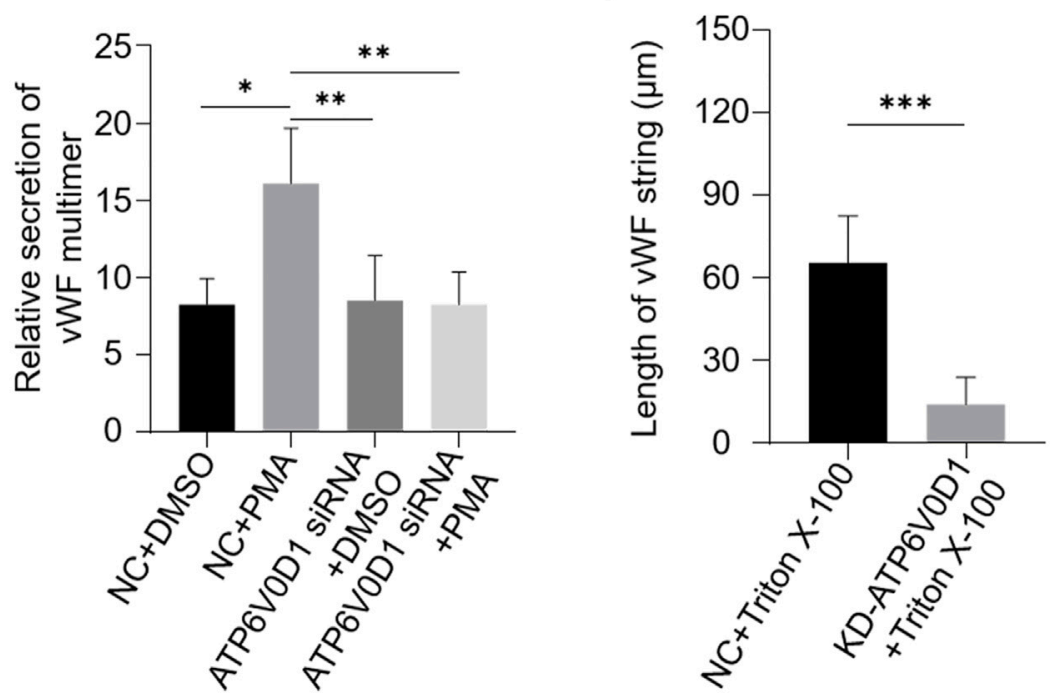

FIGURE 7 | VWF secretion and the ability to generate surface strings are also compromised in KD-ATP6VOD1 human umbilical vein endothelial cells (HUVECs). Negative control (NC) and ATP6VOD1 siRNA were transfected into two groups of HUVECs, respectively. At $72 \mathrm{~h}$ later, one group of NC and KD-ATP6V0D1 cells was exposed to $80 \mathrm{nM}$ PMA for 30 min to stimulate Weibel-Palade body secretion, and the other groups were exposed to DMSO (0.1\%) instead. (A) Western blotting analysis of the detection of ATP6VOD1 knockdown in cell lysate collection. (B, E) Western blotting analysis of the detection of vWF multimer secretion in supernatant. Multimer gels were analyzed using the $\mathrm{NIH} \mathrm{ImageJ} \mathrm{software.} \mathrm{The} \mathrm{quantification} \mathrm{of} \mathrm{supernatant} \mathrm{vWF} \mathrm{multimers} \mathrm{was} \mathrm{carried} \mathrm{out} \mathrm{based} \mathrm{on} \mathrm{the} \mathrm{normalization} \mathrm{of}$ the $\beta$-actin protein level of the cells in each well. $n=5,{ }^{*} p<0.05,{ }^{* *} p<0.01$. (C, D) $1 \%$ Triton X-100 was added into the culture medium of NC and KD-ATP6VOD1 HUVECs and cultured at $37^{\circ} \mathrm{C}$ for $1 \mathrm{~h}$. Immunofluorescence images of two groups of HUVECs labeled against vWF (green) and nucleus (DAPI, blue) were shown. Scale bar, $10 \mu \mathrm{m}$. (F) The length of VWF strings at each group of HUVECs was measured by the NIH ImageJ software $\left(n=30\right.$ per group, $\left.{ }^{\star \star \star} p<0.001\right)$. Data were expressed as mean \pm SEM. Three independent experiments were performed. 
complex that consists of two domains with 14 subunits. Except for the ATP6V0D1 subunit, HPS6 or BLOC-2 complex may be similarly involved in the trafficking of other v-ATPase subunits to WPBs. Because of the lack of available antibodies, the interactions between HPS6 and other v-ATPase subunits require further investigation. Another explanation is that the lack of one subunit destabilizes the other subunits in a heterogeneous complex. This is evident in the HPS mutants of BLOC-1/-2/-3, i.e., one subunit deficiency destabilizes the other subunits (Wei and $\mathrm{Li}, 2013$ ).

During WPB maturation, the luminal $\mathrm{pH}$ value is not constant, but it decreases gradually as the WPB matures. The direct correlation between this $\mathrm{pH}$ change and the shape of WPBs is evident by vWF multimerization and the assembly of the tubular structures. Mayadas et al. found that vWF dimers form multimers when the $\mathrm{pH}$ value is between 4.0 and 6.2, and the highest efficiency of multimerization occurs at 5.8 (Mayadas and Wagner, 1989). However, vWF multimers are no longer detectable when $\mathrm{pH}>6.2$. Using compounds such as monensin, $\mathrm{NH}_{4} \mathrm{Cl}$, and chloroquine to increase the $\mathrm{pH}$ in the medium, obvious phenotypic changes are visible under an electron microscope, and WPBs showed a significantly round shape (Michaux et al., 2006), which are consistent with the phenotypic changes of WPBs in KD-HPS6 HUVECs. This agrees with the notion that the luminal $\mathrm{pH}$ of WPBs is important for their maturation. Unfortunately, we have tested several dyes or tools to monitor the luminal $\mathrm{pH}$ changes but failed. A sensitive tool for this measurement needs to be developed.

It is known that the highly polymerized vWF polymers are stored in mature WPBs, which dock near the plasma membrane and release their contents into the blood after stimulation. Both in the endothelial cells of $r u$ mice and HUVEC cells, HPS6 deficiency not only changed the classic long rod shape of WPB but also led to a significant reduction in the number of newly generated WPBs. One possible explanation is that HPS6 may also participate in the process of WPB budding from TGN, which awaits further investigation.

In summary, we have revealed that HPS6 or BLOC-2 is involved in the trafficking of $\mathrm{v}$-ATPase subunits to maintain the acidic lumen of LROs. This provides more insights into the underlying mechanism of LRO defects and the pathogenesis of HPS.

\section{MATERIALS AND METHODS}

\section{Antibodies}

Antibodies Used in Immunofluorescence

The mouse monoclonal antibodies anti-vWF antibodies (1: 500, ab201336) were purchased from Abcam (United Kingdom). The rabbit polyclonal anti-human vWF antibodies $(1: 2,000$, A0082) were purchased from Dako (Denmark). The rabbit polyclonal anti-c-Myc antibodies (1: 500, C3956) were purchased from Sigma-Aldrich (United States). The goat anti-rabbit $\operatorname{IgG}(\mathrm{H}+\mathrm{L})$ Highly Cross-Adsorbed Secondary Antibody, Alexa Fluor 488 (1: 2,000, CA11008s) or Alexa Fluor 594 (1:2,000, CA11012s) and goat anti-mouse IgG $(\mathrm{H}+\mathrm{L})$ Highly Cross-Adsorbed
Secondary Antibody, Alexa Fluor Plus 488 (1:2,000, CA11001s) were purchased from Invitrogen (United States).

Antibodies used in Western blotting

The rabbit polyclonal anti-human vWF antibodies $(1: 10,000$, A0082) were purchased from Dako (Denmark). The rabbit polyclonal antibodies against human HPS6 (1:1,000, NBP214,100) were from Novus Biologicals (United States). The rabbit polyclonal antibodies against human ATP6V1B2 (1:500, ab73404), ATP6V1H (1:500, ab187706), and ATP6V0D1 (1: 1,000, ab202899) were all from Abcam (United Kingdom). The mouse monoclonal antibodies anti-human $\beta$-actin (1: 100,000, A5441), rabbit polyclonal anti-c-Myc antibodies (1: $1,000, \mathrm{C} 3956)$, and rabbit polyclonal anti-LAMP1 antibodies (1:2,000, L1418) were purchased from Sigma-Aldrich (United States). The mouse monoclonal anti-Flag tag (1:1,000, MA1-91878) were purchased from Invitrogen (United States). The peroxidase-conjugated secondary anti-rabbit antibodies and anti-mouse antibodies (both 1:5,000, ZB2301 and ZB2305) were all purchased from ZSGB-bio (China).

\section{Animals}

Male WT or ruby-eye ( $r u$ ) mutant (HPS6 deficiency in BLOC-2) mice ( 4 weeks of age) were used for the experiments. They were originally obtained from The Jackson Laboratory (Maine, United States) and maintained in the laboratory of Dr. Richard T. Swank. All these mutant mice arose from spontaneous mutations in $\mathrm{C} 57 \mathrm{BL} / 6 \mathrm{~J}$ background. The mutation of the Hps6 gene was confirmed by PCR analysis of tail DNA (data not shown). The animal experiments were carried out in accordance with institutional guidelines for animal experimentation, and the procedures were approved by the Institutional Animal Care and Use Committee of the Institute of Genetics and Developmental Biology, Chinese Academy of Sciences (mouse protocol \# AP2021028).

\section{Cell Culture}

Primary mouse heart endothelial cells were isolated by magnetic activated cell sorting method as previously described (Ma et al., 2016). The human umbilical vein endothelial cells were obtained from Dr. Qiang Wang's Laboratory (Institute of Zoology, Chinese Academy of Sciences) and maintained in EGM-2 medium (Lonza, cc-3162) at $37^{\circ} \mathrm{C}$ and $5 \% \mathrm{CO}_{2}$.

\section{ru EC Treatments}

Phorbol-12-myristate-13-acetate (Sigma, P1585) in DMSO was added into the EGM-2 medium with $80 \mathrm{nmol} / \mathrm{L}$ final concentration. The cells were washed with the growth medium twice after $30 \mathrm{~min}$, changing to fresh growth medium, and then fixed with $4 \%$ paraformaldehyde at $0,2,4$, and $8 \mathrm{~h}$ later, respectively.

\section{HUVEC Treatments}

PMA in $80 \mathrm{nM}$ final concentration or $0.1 \%$ DMSO was added into the EGM-2 medium (respectively) after $30 \mathrm{~min}$. The cells were washed with growth medium twice, changing to fresh growth medium, and then fixed with $4 \%$ PFA after 2, 4, 8, and $16 \mathrm{~h}$, respectively, or the supernatant was collected 
directly. Triton X-100 (Sigma-Aldrich, T8787) was used at $1 \%$ in phosphate-buffered saline (PBS) after $\mathrm{pH}$ adjustment. The Triton $\mathrm{X}$-100-treated cells were applied at $37^{\circ} \mathrm{C}$ for $1 \mathrm{~h}$. All the procedures were followed as described (Michaux et al., 2006). The v-ATPase inhibitor, bafilomycin A1 (Abcam, ab120497), in $200 \mathrm{nM}$ final concentration or $0.1 \%$ DMSO was added into the growth medium, respectively, washing the cells with growth medium twice after $1 \mathrm{~h}$ and then fixing them with 4\% PFA immediately.

\section{RNA Interference}

HPS6 expression was suppressed with a siRNA (5'-GCUGGA GAGGAAGGUCCUATT- ${ }^{\prime}$ ). ATP6V0D1 expression was suppressed with a siRNA (5'-GCA CUG AUU AUG GUA ACU UTT- $\left.3^{\prime}\right)$. The results were compared with a nontargeting NC siRNA ( $5^{\prime}$-UUCUCCGAACGUGUCACGUTT$\left.3^{\prime}\right)$. A total of $120 \mathrm{nM}$ siRNAs were introduced into HUVECs using Lipofectamine RNAiMAX transfection reagent (Thermo Fisher Scientific, 2103411) according to the protocol of the manufacturer. After transfection $(72 \mathrm{~h})$, the cells were treated as needed.

\section{Western Blotting}

Cells were lysed with cell lysis buffer for Western and IP [Beyotime, P0013, $20 \mathrm{mM}$ Tris (pH 7.5), $150 \mathrm{mM} \mathrm{NaCl,} \mathrm{1 \%}$ Triton X-100] supplemented with protease inhibitor mixture (Solarbio Life Science, P6730) on ice for $1 \mathrm{~h}$. The lysates were separated in sodium dodecyl sulfate (SDS)-PAGE gels [30\% acrylamide, 1.5 M Tris- $\mathrm{HCl}$ ( $\mathrm{pH} 8.8$ ), 1.0 M Tris- $\mathrm{HCl}$ (pH 6.8), $10 \%$ SDS, $10 \%$ ammonium persulfate, and TEMED] and then transferred to a polyvinylidene difluoride membrane (Millipore, IPVH00010), which was blocked with 5\% nonfat milk in Tris-buffered saline and $0.2 \%$ Tween-20 (TBST) for $1 \mathrm{~h}$ at room temperature, followed by overnight incubation at $4^{\circ} \mathrm{C}$ with primary antibodies. After three washes with TBST, the membrane was incubated with appropriate horseradish peroxidase-conjugated secondary antibodies for $1 \mathrm{~h}$ at room temperature. The antigen was detected with Mini Chemiluminescent Imaging and Analysis System (MiniChemi) according to the instructions of the manufacturer. The procedures were repeated with the same samples more than twice to react with each primary antibody.

\section{Immunochemistry}

Cultured cells were washed once with PBS and fixed in 4\% PFA. The cells were permeabilized with $0.4 \%$ Triton X-100 in PBS for $20 \mathrm{~min}$ and then blocked with $1 \%$ bovine serum albumin (BSA) in PBS for $2 \mathrm{~h}$ at room temperature. After blocking, the cells were incubated overnight at $4^{\circ} \mathrm{C}$ with primary antibodies against vWF (diluted 1:2,000 in 3\% BSA) and Myc tag (diluted 1:1,000 in 3\% BSA). Alexa red- or green-labeled secondary antibodies (1:2,000) were used for signal detection. To stain the nucleus, the cells were incubated with DAPI (ZSGB-BIO, ZLI-9557) before mounting. The labeled cells were observed with a confocal laser scanning microscope (ZEISS, LSM880) equipped with 405-, 488-, and 561$\mathrm{nm}$ excitation laser. Images were taken with $\times 100$ oil objective lens (Zeiss) using ZEN-Black (Zeiss).

\section{vWF Multimer Analysis}

Samples were gained from the supernatant after PMA stimulation as described above. In this experiment, the same amount of cells was seeded into each well of the 24-well plate, and the same volume of medium $(200 \mu \mathrm{l})$ was used during the PMA stimulation. After treating with $80 \mathrm{nM}$ PMA in each well for $1 \mathrm{~h}$, the supernatant samples $(150 \mu \mathrm{l})$ were loaded in $50 \mathrm{mmol} / \mathrm{L}$ Tris, $\mathrm{pH}$ 8.0, $1 \%$ SDS, 5\% glycerol, and $0.002 \%$ bromophenol blue. The vWF multimer analysis was as previously described ( $\mathrm{Ma}$ et al., 2016). Then, 1.2\% agarose gels were prepared by dissolving Seakem high-gellingtemperature agarose (Lonza, 50,041) in $0.375 \mathrm{~mol} / \mathrm{L}$ Tris ( $\mathrm{pH} 8.8$ ), with SDS added to a final concentration of $0.1 \%$. The gels were run at $30 \mathrm{~V}$ for $16 \mathrm{~h}$ (Tanon, EPS600) before transferring to a nitrocellulose membrane labeled with rabbit anti-vWF antibody (1:10,000), followed by horseradish peroxidase-conjugated anti-rabbit secondary antibody (1: 5,000 ), and developed by chemiluminescence (Meilunbio, MA0186). The multimers of each lane on the same gel were arranged according to their molecular weight. The multimer gels were analyzed using the NIH ImageJ software. The quantification of supernatant vWF multimers was carried out based on the normalization of the $\beta$-actin protein level of the cells in each well.

\section{Plasmid Transfection}

The coding sequence of ATP6V0D1 was amplified by PCR from human cDNA and cloned into the pCDNA3.1 Myc HisB vector, generating ATP6VOD1 constructs tagged with Myc at the C-terminus. The Flag-HPS6 was obtained from the lab of Jiajia Liu (Li et al., 2014). The plasmids were transfected into $293 \mathrm{~T}$ cells with Lipofectamine 2000 (Invitrogen, 11,668) according to the protocol of the manufacturer. The plasmids were transfected into HUVECs with Lipofectamine 3000 (Invitrogen, L3000-015) according to the protocol of the manufacturer.

\section{Co-immunoprecipitation Assay}

Co-IP assays were performed as described by Zhang et al. (2014). The transfected $293 \mathrm{~T}$ cells were harvested and lysed with lysis buffer (1 M Tris-HCl, pH 7.4, $5 \mathrm{M} \mathrm{NaCl}, 0.5 \mathrm{M}$ EDTA, $1 \%$ Triton $\mathrm{X}-100$, and protease inhibitors). The cell lysates were centrifuged at $12,000 \mathrm{rpm}$ for $5 \mathrm{~min}$, and the supernatant was collected and incubated overnight with anti-FLAG M2 affinity antibody (Sigma-Aldrich, A2220) at $4^{\circ} \mathrm{C}$ and washed 4 times with icecold lysis buffer. The samples were eluted with protein loading buffer (Solarbio, P1040) and subjected to SDS-PAGE and Western blotting with anti-Myc antibody $(1: 1,000)$ or anti-Flag tag antibody $(1: 1,000)$.

\section{OptiPrep Continuous Density Gradient Centrifugation}

The fractionation assay was performed using the OptiPrep gradient method as described by Zhang et al. (2014). The HUVECs were washed once with PBS and transferred to a 15$\mathrm{ml}$ centrifuge tube after digestion with trypsin. The supernatant 
was discarded after centrifugation at 1,000 rpm for $3 \mathrm{~min}$. The sample was resuspended and transferred to a 1.5-ml Eppendorf tube. The supernatant was discarded again after centrifugation. The sample was immediately homogenized with $250 \mu \mathrm{l} \mathrm{HB}$ lysis

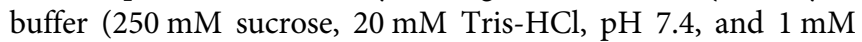
EDTA) and ground. The sample was placed onto the top of a 12.4-ml continuous 5-50\% OptiPrep (Axis-Shield, 1,114,542) gradient in $\mathrm{HB}$ buffer. The gradient was centrifuged at $30,000 \mathrm{~g}$ for $16 \mathrm{~h}$ in a Beckman SW41 rotor at $4^{\circ} \mathrm{C}$. Thirteen fractions $(900 \mu \mathrm{l}$ each) were collected from the top. Equal aliquots from each fraction were analyzed for Western blotting.

\section{Statistical Analysis}

The NIH ImageJ software was used for the quantitative analysis of Western blotting, determining the number of WPBs in a single cell, and the measurement of Feret's diameter. All results were independently repeated at least three times. All the histograms were plotted using GraphPad Prism software. Student's $t$-test was used as the statistical method, and mean \pm standard error (SEM) was used to represent the data. ${ }^{\star} p<0.05$ indicated that there were significant differences in the data. ${ }^{* *} p<0.01$ and ${ }^{* *} p<0.001$ indicated that there was a very significant difference in the data, while NS indicates that there is no significant difference in the data.

\section{REFERENCES}

Aird, W. C. (2007). Phenotypic Heterogeneity of the Endothelium. Circ. Res. 100 (2), 158-173. doi:10.1161/01.RES.0000255691.76142.4a

Arribas, M., and Cutler, D. F. (2000). Weibel-Palade Body Membrane Proteins Exhibit Differential Trafficking after Exocytosis in Endothelial Cells. Traffic 1 (10), 783-793. doi:10.1034/j.1600-0854.2000.011005.x

Dennis, M. K., Mantegazza, A. R., Snir, O. L., Tenza, D., Acosta-Ruiz, A., Delevoye, C., et al. (2015). BLOC-2 Targets Recycling Endosomal Tubules to Melanosomes for Cargo Delivery. J. Cel Biol. 209 (4), 563-577. doi:10.1083/ jcb.201410026

El-Mansi, S., and Nightingale, T. D. (2021). Emerging Mechanisms to Modulate VWF Release from Endothelial Cells. Int. J. Biochem. Cel Biol. 131, 105900. doi:10.1016/j.biocel.2020.105900

Ferraro, F., Kriston-Vizi, J., Metcalf, D. J., Martin-Martin, B., Freeman, J., Burden, J. J., et al. (2014). A Two-Tier Golgi-Based Control of Organelle Size Underpins the Functional Plasticity of Endothelial Cells. Develop. Cel 29 (3), 292-304. doi:10.1016/j.devcel.2014.03.021

Futai, M., Sun-Wada, G.-H., Wada, Y., Matsumoto, N., and Nakanishi-Matsui, M. (2019). Vacuolar-Type ATPase: A Proton Pump to Lysosomal Trafficking. Proc. Jpn. Acad. Ser. B: Phys. Biol. Sci. 95 (6), 261-277. doi:10.2183/pjab.95.018

Harrison-Lavoie, K. J., Michaux, G., Hewlett, L., Kaur, J., Hannah, M. J., LuiRoberts, W. W. Y., et al. (2006). P-Selectin and CD63 Use Different Mechanisms for Delivery to Weibel-Palade Bodies. Traffic 7 (6), 647-662. doi:10.1111/j.1600-0854.2006.00415.x

Karampini, E., Bierings, R., and Voorberg, J. (2020). Orchestration of Primary Hemostasis by Platelet and Endothelial Lysosome-Related Organelles. Arterioscler Thromb. Vasc. Biol. 40 (6), 1441-1453. doi:10.1161/ATVBAHA. 120.314245

Li, K., Yang, L., Zhang, C., Niu, Y., Li, W., and Liu, J.-J. (2014). HPS6 Interacts with Dynactin p150Glued to Mediate Retrograde Trafficking and Maturation of Lysosomes. J. Cel Sci. 127 (21), 4574-4588. doi:10.1242/jcs.141978

Lui-Roberts, W. W. Y., Collinson, L. M., Hewlett, L. J., Michaux, G., and Cutler, D. F. (2005). An AP-1/Clathrin Coat Plays a Novel and Essential Role in Forming the Weibel-Palade Bodies of Endothelial Cells. J. Cel Biol. 170 (4), 627-636. doi:10.1083/jcb.200503054

\section{DATA AVAILABILITY STATEMENT}

The raw data supporting the conclusion of this article will be made available by the authors without undue reservation.

\section{AUTHOR CONTRIBUTIONS}

WL and JM designed the study and administrated the whole study. JL and JM conducted the experiments, collected the data, and drafted the manuscript. ZH was involved in methodology and statistical analysis. All authors gave final approval of the version to be published and agreed to be responsible for all aspects of this work.

\section{FUNDING}

This work was supported by grants from the Ministry of Science and Technology of China (2019YFA0802104 to WL), from the National Natural Science Foundation of China (91854110 to ZH and 31830054 to WL), from Beijing Natural Science Foundation (5192008 to JM), and from Beijing Municipal Hospitals Scientific Research and Cultivation Program (PX2018048 to JM).

Ma, J., Zhang, Z., Yang, L., Kriston-Vizi, J., Cutler, D. F., and Li, W. (2016). BLOC-2 subunit HPS6 deficiency affects the tubulation and secretion of von Willebrand factor from mouse endothelial cells. J. Genet. Genomics 43 (12), 686-693. doi:10.1016/j.jgg.2016.09.007

Marks, M. S., Heijnen, H. F., and Raposo, G. (2013). Lysosome-Related Organelles: Unusual Compartments Become Mainstream. Curr. Opin. Cel Biol. 25 (4), 495-505. doi:10.1016/j.ceb.2013.04.008

Mayadas, T. N., and Wagner, D. D. (1989). In Vitro Multimerization of von Willebrand Factor is Triggered by Low pH. J. Biol. Chem. 264 (23), 13497-13503. doi:10.1016/s0021-9258(18)80024-2

Mazzotta, C., Marden, G., Farina, A., Bujor, A., Trojanowski, M. A., and Trojanowska, M. (2020). FLI1 and ERG Protein Degradation Is Regulated via Cathepsin B Lysosomal Pathway in Human Dermal Microvascular Endothelial Cells. Microcirculation 28 (1), e12660. doi:10.1111/micc.12660

McCormack, J. J., Lopes Da Silva, M., Ferraro, F., Patella, F., and Cutler, D. F. (2017). Weibel-Palade Bodies at a Glance. J. Cel Sci. 130 (21), 3611-3617. doi: $10.1242 /$ jcs. 208033

Michaux, G., Abbitt, K. B., Collinson, L. M., Haberichter, S. L., Norman, K. E., and Cutler, D. F. (2006). The Physiological Function of von Willebrand's Factor Depends on its Tubular Storage in Endothelial Weibel-Palade Bodies. Develop. Cel 10 (2), 223-232. doi:10.1016/j.devcel.2005.12.012

Michaux, G., and Cutler, D. F. (2004). How to Roll an Endothelial Cigar: The Biogenesis of Weibel-Palade Bodies. Traffic 5 (2), 69-78. doi:10.1111/j.16000854.2004.00157.x

Olmedo, I., Pino, G., Riquelme, J. A., Aranguiz, P., Díaz, M. C., López-Crisosto, C., et al. (2020). Inhibition of the Proteasome Preserves Mitofusin-2 and Mitochondrial Integrity, Protecting Cardiomyocytes during IschemiaReperfusion Injury. Biochim. Biophys. Acta (Bba) - Mol. Basis Dis. 1866 (5), 165659. doi:10.1016/j.bbadis.2019.165659

Savage, B., Sixma, J. J., and Ruggeri, Z. M. (2002). Functional Self-Association of von Willebrand Factor during Platelet Adhesion under Flow. Proc. Natl. Acad. Sci. 99 (1), 425-430. doi:10.1073/pnas.012459599

Schillemans, M., Karampini, E., Kat, M., and Bierings, R. (2019). Exocytosis of Weibel-Palade Bodies: How to Unpack a Vascular Emergency Kit. J. Thromb. Haemost. 17 (1), 6-18. doi:10.1111/jth.14322

Tang, T., Xu, W., Ma, J., Wang, H., Cui, Z., Jiang, T., et al. (2019). Inhibitory Mechanisms of DHA/CQ on pH and Iron Homeostasis of Erythrocytic Stage 
Growth of Plasmodium Falciparum. Molecules 24 (10), 1941. doi:10.3390/ molecules 24101941

Tiemeier, G. L., Koning, R., Wang, G., Kostidis, S., Rietjens, R. G. J., Sol, W. M. P. J., et al. (2020). Lowering the Increased Intracellular $\mathrm{pH}$ of Human-Induced Pluripotent Stem Cell-derived Endothelial Cells Induces Formation of Mature Weibel-Palade Bodies. STEM CELLS Transl Med. 9 (7), 758-772. doi:10.1002/ sctm.19-0392

Valentijn, K. M., Valentijn, J. A., Jansen, K. A., and Koster, A. J. (2008). A New Look at Weibel-Palade Body Structure in Endothelial Cells Using Electron Tomography. J. Struct. Biol. 161 (3), 447-458. doi:10.1016/j.jsb.2007. 08.001

Wagner, D. D., Olmsted, J. B., and Marder, V. J. (1982). Immunolocalization of von Willebrand protein in Weibel-Palade bodies of human endothelial cells. J. Cel Biol. 95 (1), 355-360. doi:10.1083/jcb.95.1.355

Warhol, M. J., and Sweet, J. M. (1984). The ultrastructural localization of von Willebrand factor in endothelial cells. Am. J. Pathol. 117 (2), 310-315.

Wei, A.-H., and Li, W. (2013). Hermansky-Pudlak Syndrome: Pigmentary and Non-Pigmentary Defects and Their Pathogenesis. Pigment Cel Melanoma Res 26 (2), 176-192. doi:10.1111/pcmr.12051

Weibel, E. R., and Palade, G. E. (1964). New Cytoplasmic Components in Arterial Endothelia. J. Cel Biol. 23 (1), 101-112. doi:10.1083/jcb.23.1.101
Zhang, Z., Hao, C.-J., Li, C.-G., Zang, D.-J., Zhao, J., Li, X.-N., et al. (2014). Mutation of SLC35D3 Causes Metabolic Syndrome by Impairing Dopamine Signaling in Striatal D1 Neurons. Plos Genet. 10 (2), e1004124. doi:10.1371/ journal.pgen.1004124

Conflict of Interest: The authors declare that the research was conducted in the absence of any commercial or financial relationships that could be construed as a potential conflict of interest.

Publisher's Note: All claims expressed in this article are solely those of the authors and do not necessarily represent those of their affiliated organizations or those of the publisher, the editors, and the reviewers. Any product that may be evaluated in this article or claim that may be made by its manufacturer is not guaranteed or endorsed by the publisher.

Copyright $\odot 2022 \mathrm{Lu}, \mathrm{Ma}, \mathrm{Hao}$ and Li. This is an open-access article distributed under the terms of the Creative Commons Attribution License (CC BY). The use, distribution or reproduction in other forums is permitted, provided the original author(s) and the copyright owner(s) are credited and that the original publication in this journal is cited, in accordance with accepted academic practice. No use, distribution or reproduction is permitted which does not comply with these terms. 